Proceedings of the Contemporary Ergonomics and Human Factors 2015:

International Conference on Ergonomics \& Human Factors,

13-16 April 2015, Daventry, UK.

\title{
THE CASE FOR THE DEVELOPMENT OF NOVEL HUMAN SKILLS CAPTURE METHODOLOGIES
}

\author{
Everitt, J. W., Fletcher, S. R.
}

Industrial Psychology and Human Factors Group, Cranfield University, UK

\begin{abstract}
As the capabilities of industrial automation are growing so is the ability to supplement or replace the more tacit, cognitive skills of manual operators. Whilst models have been published within the human factors literature regarding automation implementation they neglect to discuss the initial capture of the task and automation experts currently lack a formal tool to assess feasibility. The definition of what is meant by "human skill" is discussed and three crucial theoretical underpinnings are proposed for a novel, automation-specific skill capture methodology: emphasis upon procedural rules, emphasis upon action-facilitating factors and taxonomy of skills.
\end{abstract}

\section{Introduction}

The capability of automation to replace or supplement human activity in manufacturing is increasing, however there exists no method to decide what tasks are suitable for intelligent automation and to what extent should they be automated. Often the default strategy taken by system designers is to simply automate all the functions that it is economically viable and technically possible to do so. Typically, the human is still required for task performance involving 'fiddly' manual dexterity or which requires cognitive reasoning and decisionmaking. However, this often leaves the human operator to 'pick up the slack' and perform the functions that the system designer could not automate; thus the human operators' role is defined not by the optimisation of the entire humanmachine system but rather by the creativeness (or lack thereof) of the system designer (Parasuraman, Sheridan and Wickens, 2000). This disregard for the abilities, limitations and needs of the human operator can lead to a range of human factors issues such as loss of skills, operator complacency and reduced situational awareness (Endsley and Kiris, 1995).

A number of papers have been published discussing the process of automation implementation and function allocation with an eye on both operator well-being and system reliability (Endsley and Kaber, 1999; Kaber and Endsley, 2004; Lin, Yen and Yang, 2010; Parasuraman et al, 2000). Notably the initial capture of the task is not discussed by these studies, perhaps under the assumption that traditional methods such as Hierarchical Task Analysis (HTA) are suitable for 
function allocation (Marsden and Kirby, 2004). Whilst in the past these methods may have been sufficient for the automation of simpler, less cognitively demanding tasks, but more tacit and complex cognitive factors must be accounted for during the implementation of intelligent automation. Effective automation strategy depends upon accurate and sufficiently detailed decomposition of tasks, thus one of the challenges of effective intelligent automation in manufacturing is being able to accurately capture both the implicit and tacit skills deployed by human operators during task completion, both physical and cognitive.

From a mechatronic perspective, experts in that field are better suited to determine the feasibility of automation based upon technical possibility and / or cost effectiveness, however human factors is ideally placed to inform and advise these decisions if it can offer a detailed, accurate account of the physical, perceptual and cognitive processes that facilitate skilled behaviour. A detailed account of the human faculties should assist system designers to identify analogies between human processes and potential automated solutions which mimic human performance, but also devise ways in which automation may circumvent the required faculty.

In brief, human factors and automation engineers currently lack a formal methodology to fully evaluate the suitability of high skill manual tasks for automation. This paper aims to outline why a novel skill capture methodology is needed for intelligent automation implementation and the required theoretical underpinnings. In order to accomplish this we first need to understand what is meant by 'skill'.

\section{Defining skill}

Human skill can apply to a broad spectrum of behaviours, for example both an expert cellist and a grand master chess player could be considered highly skilled in their respective domains, however playing the cello requires precise motor actions of the fingers and upper limbs, while all the of the 'skill' which differentiates an expert from a novice chess player is purely cognitive; an expert is no better than a novice at physically moving a piece. Skill is therefore best considered an umbrella term for a range of abilities, and thus a comprehensive understanding of human skill must recognise and effectively discriminate between different types of skilled behaviour as well as consider their interactions.

To understand what is meant by 'skill' is to define skilled behaviour from unskilled behaviour. Proctor and Dutta (1995) defined human skill as "goal directed', well organised behaviour that is acquired through practice and performed with economy of effort" (p18). This definition posits four characteristics of human performance which define it as skillful; the first characteristic is 'goal directed', it could be argued is not exclusive to skilled performance as unskilled performance can also be goal directed and as such this 
exists as a pre-condition rather than a defining feature of skillful behaviour. This then places an emphasis on the economical, well organised, and acquired by practice characteristics which defines skillful behaviour from unskillful.

Another perspective is offered by Rosenbaum, Carlson and Gilmore (2001) who stated that skill is "an ability that allows a goal to be achieved within some domain with increasing likelihood as a result of practice" (p454). As with Proctor and Dutta (1995), goal direction is noted as well as acquisition through practice. However instead of the emphasis being placed upon the efficiency of actions instead skilled performance is defined by the likelihood of success. This is not a trivial distinction, the latter definition allows inefficient but successful practices to be labeled as skillful whilst the former argues that it is increasing efficiency through practice which defines the acquisition of skill and that the likelihood of success is less relevant.

It is likely that the validity to both assertions is to varying extents dependent upon the task; for example if completion of task by a novice is likely regardless of time taken or errors made then the skilled component of the behaviour is better defined by Proctor and Duttas' (1995) definition based on efficiency. However, if the task is unlikely to be completed by a novice (e.g. trying to defeat an expert chess player) then Rosenbuam et al's (2001) definition based upon likelihood of success is more appropriate. Most real world tasks are unlikely to be arbitrarily one or the other; instead most tasks are composed of both characteristics in varying proportions.

Both definitions agree that skill is acquired through practice; thus it can be inferred that the improvement of task performance related to practice is the key defining feature of skillful behaviour and that improvement is defined by the context and features of the task, rather than by arbitrary declaration. Although it could be argued that this simpler definition is too reductionist, as discussed, the term skill is an umbrella term for a broad range of behaviour rather than a precise definition due to the vast differences in what can be considered 'skilled'.

\section{Human skills capture}

Within a manufacturing context the view of human skills being defined by the practice placed upon them holds extra significance when one considers the nature of manufacturing tasks: being predictable, occurring in stable work environments, and being oft practiced and repeated. Thus it could be speculated that within manufacturing operators rarely have to strategise independently or create novel solutions but rather operators actions are governed by predetermined procedures, i.e. the tacit skills in manufacturing are more like the cellist's than the chess player's. Thus the key to capturing tacit human skills in manufacturing is to capture the tacit 'unofficial' procedures which govern skilled behaviour. In turn effective skills capture methodologies should assume a procedural nature of the task and emphasise the capture these tacit procedural rules used by operators in task performance. 
It could be argued that current methods of task analysis such as Hierarchical Task Analysis (HTA) (Annett, 2003; Annett and Duncan, 1967) would be sufficient to capture the tacit procedural rules deployed by operators during task performance. HTA's emphasis is upon the identification of task goals and subgoals to create a nested hierarchy. This nested hierarchy is then furnished with 'operations' and 'plans' to record the actions being undertaken and how these operations are ordered respectively (Stanton, 2006). Whilst HTA has been demonstrated to assist automation implementation (Tan, Duan, Zhang, and Arai, 2008) it does not account for more cognitive elements (Phipps, Meakin and Beatty, 2011) and in particular action-facilitating factors like visual judgement and haptic feedback. Whilst these factors may be superfluous for simple tasks, when considering high level, tacit skills these factors must be accounted for in order to achieve a comprehensive account of the task and how optimal performance is achieved.

Finally, Bullock, Ma and Dollar (2013) demonstrated the effectiveness of standardised taxonomy of fine motor movements for the transfer of simple tasks, such as picking up a coin. It is posited that a novel, more comprehensive taxonomy of skills which encompassed all physical, perceptual, and cognitive faculties could be used to identify analogies between human operations and potential automated solutions. The identification and classification of the human faculties which facilitate human performance would allow automation and human factors experts to analyse each individual sub-task for feasibility of automation and assess the impact of automation upon the entire task.

\section{Conclusions}

This paper has highlighted three major requirements for an effective human skills capture methodology to assess the feasibility of automation: an emphasis upon capturing the tacit procedures, an emphasis upon capturing the actionfacilitating factors, and taxonomy of skill types. The specificity of the requirements are such that novel analytical tools are necessary to keep pace with the growing capabilities of intelligent automation.

At the time writing a novel skills capture methodology is currently under development which seeks to encompass the three major requirements; Discrete Task Analysis (DTA). Instead of replacing traditional methods such as HTA, DTA is a Task Decomposition based theoretical framework intended to supplement HTA by capturing physical manipulations and, perceptual and cognitive action-facilitating factors within the context of specifically devised skills taxonomy. DTA's development has been grounded in industrial case studies in tasks such as aircraft assembly and steel polishing. 


\section{References}

Annett, J. 2003. Hierarchical task analysis. In Hollnagal, E. (Ed.), Handbook of cognitive task design, (CRC Press), 17-35Annett, J. and Duncan, K. D. 1967, "Task analysis and training design", Occupational Psychology 41, 211-221.

Bullock, I. M., Ma, R. R. and Dollar, A. M. 2013, "A hand-centric classification of human and robot dexterous manipulation", IEEE transactions on haptics 6(2) $129-144$.

Endsley, M. R. and Kaber, D. B. 1999, "Level of automation effects on performance, situation awareness and workload in a dynamic control task", Ergonomics. 42(3), pp. 462-492.

Endsley, M. R. and Kiris, E. O. 1995, "The out-of-the-loop performance problem and level of control in automation", Human factors 37(2): 381-394.

Kaber, D. B. and Endsley, M. R. 2004, "The effects of level of automation and adaptive automation on human performance, situation awareness and workload in a dynamic control task", Theoretical Issues in Ergonomics Science 5(2): 113-153.

Lin, C. J., Yenn, T. and Yang, C. 2010, "Optimizing human-system interface automation design based on a skill-rule-knowledge framework", Nuclear Engineering and Design 240(7): 1897-1905.

Parasuraman, R., Sheridan, T. B. and Wickens, C. D. (2000), "A model for types and levels of human interaction with automation", IEEE Transactions on Systems, Man, and Cybernetics Part A:Systems and Humans 30(3): 286-297.

Phipps, D. L, Meakin, G. H. and Beatty, P. C. W. 2011. "Extending hierarchical task analysis to identify cognitive demands and information design requirements" Applied Ergonomics, 42 741-748

Proctor, R. W., and Dutta, A. 1995, Skill acquisition and human performance. (Sage Publications, England)

Rosenbaum, D. A., Carlson, R. A., \& Gilmore, R. O. 2001, "Acquisition of intellectual and perceptual-motor skills". Annual Review of Psychology 52(1) 453-470.

Stanton, N. A. 2006, "Hierarchical task analysis: Developments, applications, and extensions", Applied Ergonomics 37( 1) 55-79.

Tan, J. T. C., Duan, F., Zhang, Y., and Arai, T. 2008. "Task Decomposition of Cell Production Assembly Operation for Man-Machine Collaboration by HTA" Proceedings of the IEEE International Conference on Automation and Logistics 1066-1071 
2015-04-13

\section{The case for the development of novel human skills capture methodologies}

Everitt, Jamie

Taylor \& Francis

Everitt, J., Fletcher, SR. The case for the development of novel human skills capture methodologies, Proceedings of the Contemporary Ergonomics and Human Factors 2015: International Conference on Ergonomics \& Human Factors, 13-16 April 2015, Daventry, UK http://www.crcnetbase.com/doi/abs/10.1201/b18293-59

Downloaded from Cranfield Library Services E-Repository 